\title{
Association of benzodiazepine and Z-drug use with the risk of hospitalisation for fall- related injuries among older people: a nationwide nested case-control study in Taiwan
}

Nan-Wen Yu ${ }^{1,2}$, Pei-Jung Chen ${ }^{1}$, Hui-Ju Tsai ${ }^{3,4}$, Chih-Wan Huang ${ }^{1,2}$, Yu-Wen Chiu' ${ }^{1,2}$, Wen-Ing Tsay ${ }^{5}$, Jui Hsu ${ }^{5}$ and Chia-Ming Chang ${ }^{1,2^{*}}$ (D)

\begin{abstract}
Background: Non-benzodiazepine hypnotics (Z-drugs) are advocated to be safer than benzodiazepines (BZDs). This study comprehensively investigated the association of BZD and Z-drug usage with the risk of hospitalisation for fall-related injuries in older people.

Methods: This study used the Taiwan National Health Insurance Database with a nested matched case-control design. We identified 2238 elderly patients who had been hospitalised for fall-related injuries between 2003 and 2012. They were individually matched (1:4) with a comparison group by age, sex, and index year. Conditional logistic regression was used to determine independent effects of drug characteristics (type of exposure, dosage, half-life, and polypharmacy) on older people.

Results: Older people hospitalisation for fall-related injuries were significantly associated with current use of BZDs (adjusted odds ratio $[A O R]=1.32,95 \%$ confidential interval $[\mathrm{Cl}]=1.17-1.50)$ and $Z$-drugs $(A O R=1.24,95 \% \mathrm{Cl}=1$. 05-1.48). At all dose levels of BZDs, high dose levels of Z-drugs, long-acting BZD, and short-acting BZD use were all significantly increased the risk of fall-related injuries requiring hospitalisation. Polypharmacy, the use of two or more kinds of BZDs, one kind of BZD plus Z-drugs and two or more kinds of BZDs plus Z-drugs, also significantly increased the risk $(A O R=1.61,95 \% \mathrm{Cl}=1.38-1.89 ; A O R=1.65,95 \% \mathrm{Cl}=1.08-2.50$, and $\mathrm{AOR}=1.58,95 \% \mathrm{Cl}=1.21-2.07)$.
\end{abstract}

Conclusions: Different dose levels and half-lives of BZDs, a high dose of Z-drugs, and polypharmacy with BZDs and Z-drugs were associated with an increased risk of fall-related injury requiring hospitalisation in older people. Physicians should balance the risks and benefits when prescribing these drug regimens to older people considering the risk of falls.

Keywords: Benzodiazepine, Z-drugs, Older people, Fall, Hospitalisation

\footnotetext{
* Correspondence: cmchang581@gmail.com

${ }^{1}$ Department of Psychiatry, Chang Gung Memorial Hospital at Linkou and

Chang Gung University, Taoyuan, Taiwan

${ }^{2}$ Division of Rehabilitation \& Community Psychiatry, Department of

Psychiatry, Chang Gung Memorial Hospital at Taoyuan, Taoyuan, Taiwan

Full list of author information is available at the end of the article
} 


\section{Background}

Falls are common in older people and are the second leading cause of accidental or unintentional deaths worldwide [1]. Approximately $28 \%-35 \%$ of people aged 65 years and older fall each year [2], and a study found that fall-related trauma accounts for $5.3 \%$ of all hospitalisations in this age range [3]. The risk of falls is multifactorial, and medications are modifiable extrinsic risk factors $[4,5]$. Meta-analyses and systematic reviews have reported that some classes of medications, such as benzodiazepines (BZDs), increase the risk of falls in older people [6-8].

BZDs are among the most prescribed psychotropic medications, especially in older people [9]. Because BZDs can be used as sedatives and hypnotics, they can cause problems as dependence and abuse [10] besides the side effects of dizziness, drowsiness, and coordination impairment. Moreover, after controlling the confounding factors, several studies have reported that BZDs independently increase the risk of accidents such as falls $[11,12]$, hip fractures $[13,14]$, and motor vehicle accidents [15]. However, data on whether different characteristics (as exposure duration, daily dose, and elimination half-life) lead to different risks are inconsistent. A study reported that the use of long-acting BZDs increases the risk of falls [16], whereas other studies have reported that the use of short-acting BZDs also increases this risk [17, 18]. In addition, another study indicated that dosage contributes more to the risk of falls than elimination half-life [19].

Z-drugs (zolpidem, zopiclone, and zaleplon) are non-BZD hypnotics and are advocated to be safer than BZDs [20]. Thus, the prescription of Z-drugs has been increasing rapidly [21]. A study reported that the annual use of Z-drugs in older people doubled from 2001 to 2010 in Taiwan [22]. However, some studies have demonstrated that Z-drugs also cause problems of abuse and dependence [23], and increase the risk of falls [24] and hip fractures [25].

Polypharmacy is an arising issue in public health and has a crucial role as a risk factor for falls [26]. Studies have found that the simultaneous use of two or more anxiolytics or hypnotics (another kind of polypharmacy) is common in Taiwan [27]. However, whether such polypharmacy with BZDs and Z-drugs increases the risk of fall-related injuries requiring hospitalisation in Taiwan remains unknown.

Since it was found that BZDs and zolpidem (one kind of Z-drugs) were two of the top five reported abused drugs by medical institutions in Taiwan [28] and that their long-term use (defined as 180 prescription days within a year) was not uncommon [29], it is important to understand their potential risk. This study investigated the association of the use of BZDs and Zdrugs with the risk of hospitalisation for fall-related injuries, with a focus on exposure duration, daily dose, elimination half-life, and BZD and Z-drug polypharmacy in older people.

\section{Methods}

Data sources

This is a nested matched case-control study. We obtained data from the National Health Insurance Research Database (NHIRD) provided by the Ministry of Health and Welfare (MHW) in Taiwan. The Taiwanese government launched the National Health Insurance (NHI) programme in March 1995; the NHI programme covered more than $99 \%$ of the total population of Taiwan by the end of 2008 [30]. The NHIRD was developed at the National Health Research Institutes, which linked data from demographic and enrolment records, hospital claims, ambulatory care visits, and pharmacydispensing claims from hospitals, outpatient clinics, and community pharmacies. Every person in Taiwan has a unique personal identification number. To secure patients' confidentiality, the MHW removed all identifiable patient information from the NHIRD. In this study, we used a subset of the NHIRD: Longitudinal Health Insurance Database 2000 (LHID 2000). The LHID 2000 is a data set released by the NHIRD that contains all original claims data for 200 thousand randomly selected beneficiaries in the 2000 Registry of Beneficiaries. The sex and age distributions in the sample were not significantly different from those in the general population.

Our study was approved by the Institutional Review Board of Chang Gung Memorial Hospital (No.1036020B). No informed consent was required from the subjects because the data were analysed anonymously. We analysed data from 2003 to 2012. There were no BZD and Z-drug prescription policy changes during the study period.

\section{Study population \\ Definition of cases of hospitalisation for fall-related injuries}

Between 2003 and 2012, the Taiwan National Health Insurance System still used the International Classification of Diseases, Ninth Revision, Clinical Modification (ICD9-CM) codes, and each inpatient has up to 5 discharge codes. The incident cases of hospitalisations for fallrelated injuries were defined as subjects aged 65 years and older with discharge diagnosis codes between E-880 and E-888 from 2003 to 2012. The admission date of fall-related hospitalisation was defined as the index date.

\section{Definition of the comparison group}

The comparison group consisted of subjects randomly selected from the remaining study population who did 
not have any record of discharge diagnosis of fall-related injury during 2003-2012. These subjects were individually matched with the case subjects according to sex, birth year, and index year at a ratio of 1:4. Each comparison subject was assigned an index date that matched one of the case subjects. If there were less than 4 eligible subjects, they were all included.

Initially we identified 2324 eligible case subjects. To avoid missing information, we excluded 86 cases who could not match any controls. As a result, we therefore included 2238 cases and 8645 matched controls (3.86 controls per case) in the subsequent analysis. A detailed flow diagram for identifying the study cohort is showed in Fig. 1.

\section{Definition of BZD and Z-drug usage} Types of BZD and Z-drug exposure

In Taiwan, all the BZDs and Z-drugs are only available with prescriptions from physicians. Following the classification of Ray et al. [31], the types of BZD and Z-drug exposure were classified based on when the most recent fill prescription during the 365 days before the index date and were divided into three groups: 1-30 (current users), 31-90 (indeterminate users), and 91-365 days (former users), respectively. BZD fills on the day of the hospitalisation date were excluded to avoid the problems in the temporality of the exposure-outcome. We defined the group without any BZD or Z-drug prescription within 365 days before the index date as "non-users" group.

\section{Dose of BZD and Z-drugs}

We calculated the daily dose of BZDs and Z-drugs as a defined daily dose (DDD) of diazepam $(1 \mathrm{DDD}=10 \mathrm{mg})$ for all BZDs and Z-drug prescriptions that overlapped with the index date. We used the WHOCC Index [32] (defined as ATC/DDD) as reference. Based on the distribution of the doses, the subjects were divided into the following three groups: low $(<0.3)$, medium $(0.3-0.6)$, and high (>0.6) DDD [13].

\section{Elimination half-life of BZDs and Z-drugs}

Diazepam, flurazepam, chlordiazepoxide, and clonazepam were classified as BZDs with long half-life (over $24 \mathrm{~h}$ ). The other BZDs (alprazolam, bromazepam, brotizolam, clobazam, clorazepate, cloxazolam, estazolam, fludiazepam, flunitrazepam, lorazepam, lormetazepam,

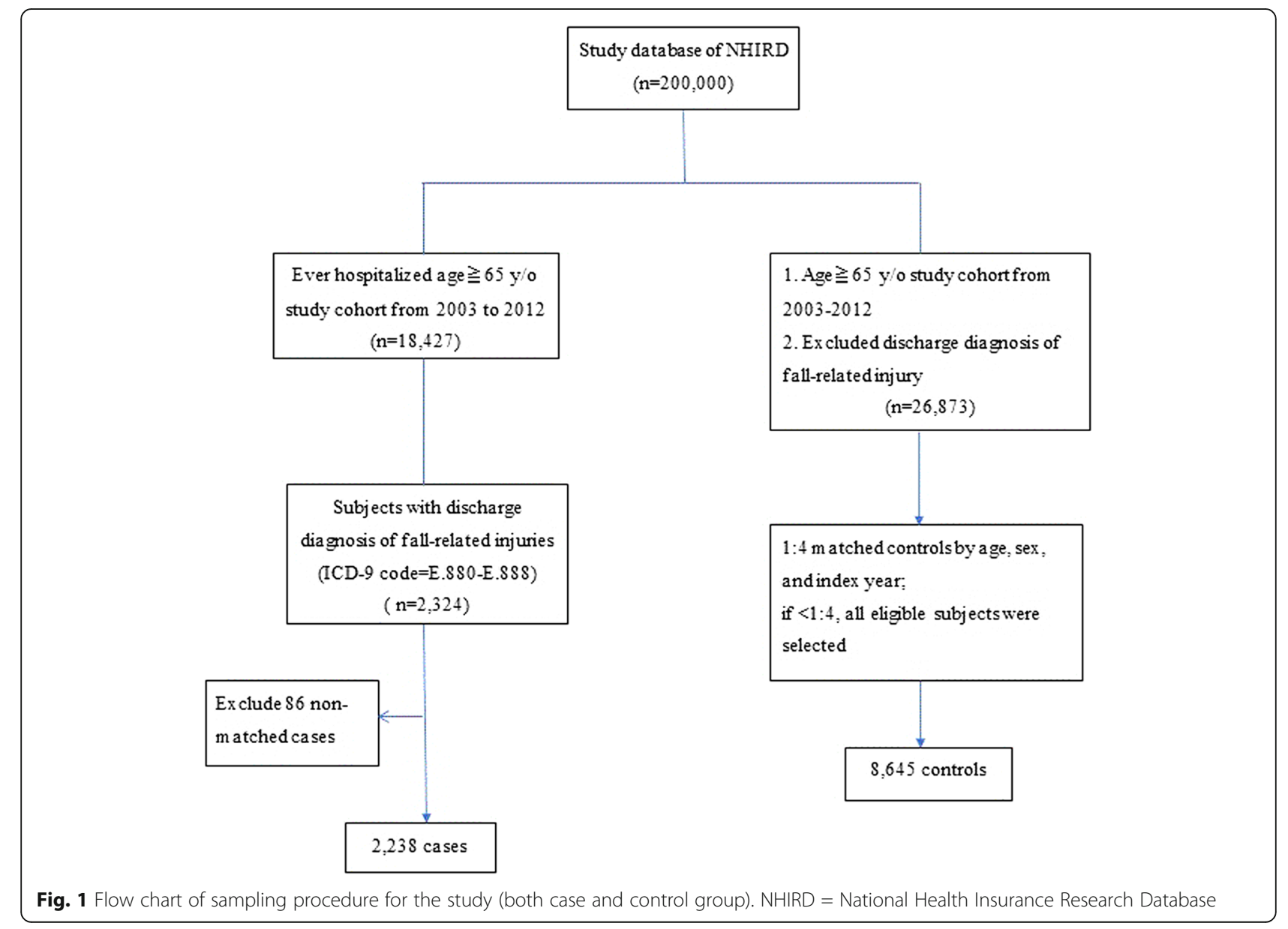


medazepam, midazolam, nimetazepam, nitrazepam, nordazepam, oxazepam, oxazolam, prazepam, temazepam, and triazolam) were classified as short half-life agents (less than $24 \mathrm{~h}$ ).

\section{Polypharmacy with BZDs and Z-drugs}

According to Wang et al. [27], polypharmacy was defined as exposure to two or more BZDs or any BZDs plus Z-drugs within 30 days before the index date. Since the combination of two or more Z-drugs is very rare, we did not analyze such combinations as polypharmacy.

\section{Covariates}

We used the outpatient claims to identify potential confounders including dementia (ICD-9-CM code 290*), epilepsy (ICD-9-CM 345*), Parkinson's disease (ICD-9CM 332*), cerebrovascular disease (ICD-9-CM 430*438*), diabetes (ICD-9-CM 250*), hypertension (ICD-9CM $401^{*}-405^{*}$ ), and ischemia heart disease (ICD-9-CM $\left.410^{*}-415^{*}\right)$. All prescriptions filled during the 365 days prior to the index date were reviewed to verify the inclusion of antipsychotics, antidepressants, anticonvulsants, thiazide diuretics, and opioids. In addition, we calculated the Charlson comorbidity index scores (CCI) [33] based on the comorbid diagnoses and number of outpatient services received during the 6 months before the index date. The CCI was developed, originally, as a prognostic indicator for patients with a variety of medical conditions and has been commonly used to measure patients' comorbid conditions.

\section{Statistical analysis}

We first compared the demographic characteristics, comorbidities, exposure to other medications, and health care utilisation between the case and comparison subjects. The unadjusted odds ratio was calculated through bivariate conditional logistic regression. Multivariate analysis was performed using conditional logistic regression to determine the independent effects of BZD and Z-drug characteristics (e.g., type of exposure, dosage, half-life, and polypharmacy) on older people. Multivariate results are reported as adjusted odds ratio (AOR) with 95\% confidence interval. Analyses were performed using SAS, version 6.0 (SAS Institute, Inc., Cary, NC).

\section{Results}

The demographic and clinical characteristics of the case and comparison subjects are listed in Table 1 . The mean age of the fall patients was 77.7 years, and $60.7 \%$ were women. Compared with the control subjects, the case subjects were more likely to have been diagnosed with dementia, Parkinson's disease, and cerebrovascular disease while controls were more likely to have been diagnosed of hypertension and ischemia heart disease during the 365 days before the index date. In addition, the case subjects were more frequently exposed to antipsychotics, antidepressants, and opioids but less likely to exposed to thiazide diuretics during this study period. Compared with the control subjects, the case subjects had significantly less received outpatient services in the 180 days before the index date.

Table 2 illustrates the relationship between the risk of hospitalisation for fall-related injuries and the types of exposure to BZDs and Z-drugs. The current users of BZDs were significantly associated with a high risk of hospitalisation for fall-related injuries after adjustment for covariates $(\mathrm{AOR}=1.32,95 \% \mathrm{CI}=1.17-1.50)$. However, the risk was lower in the indeterminate and former users of BZDs than in the nonusers (AOR $=0.75,95 \%$ $\mathrm{CI}=0.62-0.91$ for indeterminate users and $\mathrm{AOR}=0.74$, $95 \% \mathrm{CI}=0.64-0.86$ for former users). Compared with Z-drug non-users, the current users of Z-drugs also showed significantly higher risk of hospitalisation for fall-related injuries after adjusting the covariates (AOR $=1.24,95 \% \mathrm{CI}=1.05-1.48$ ).

Table 3 demonstrates the relationship between the risk of hospitalisation for fall-related injuries and the dose levels of BZDs and Z-drugs among the current users. After adjustment for covariates, significantly increased AORs were observed for all dose levels of BZDs users $(\mathrm{AOR}=1.75,95 \% \mathrm{CI}=1.47-2.08$; $\mathrm{AOR}=1.54,95 \% \mathrm{CI}=1.28-1.85 ;$ and $\mathrm{AOR}=1.27$, $95 \% \mathrm{CI}=1.08-1.50$, respectively, for high, medium, and low dose levels). For the current users of Zdrugs, only the high dose level significantly increased the risk after adjusting the covariates $(\mathrm{AOR}=1.37$, 95\% CI $=1.14-1.64$ ).

Table 4 shows the relationship between the risk of hospitalisation for fall-related injuries and the characteristics of BZD exposure with respect to the index date, the elimination half-life, and polypharmacy. For the elimination half-life, all three categories, namely long-acting BZDs $(\mathrm{AOR}=1.41,95 \% \mathrm{CI}=1.16-1.71)$, short-acting BZDs $(\mathrm{AOR}=1.42,95 \% \mathrm{CI}=1.20-1.69)$, and combined long- and short-acting BZDs $(\mathrm{AOR}=1.61,95 \% \mathrm{CI}=1.37-1.89)$, significantly increased the risk after adjustment for covariates. Compared with nonusers, treatment with only one kind of BZD and only Z-drugs were associated increased risk of fall-related injury $(\mathrm{AOR}=1.40,95 \%$ $\mathrm{CI}=1.19-1.65$ and $\mathrm{AOR}=1.33,95 \% \mathrm{CI}=1.04-1.69$ ). Regarding polypharmacy, two or more types of BZD $(\mathrm{AOR}=1.61,95 \% \mathrm{CI}=1.38-1.89)$, one kind of $\mathrm{BZD}$ + Z-drugs $(\mathrm{AOR}=1.65,95 \% \mathrm{CI}=1.08-2.50)$, and two or more types of BZD plus Z-drugs $(\mathrm{AOR}=1.33$, 95\% CI $=1.04-1.69)$ significantly increased the risk of hospitalisation for fall-related injuries. The risk of fall-related injury requiring hospitalisations when 
Table 1 Demographic and clinical characteristics of the elderly patients hospitalised for fall injuries

\begin{tabular}{|c|c|c|c|c|c|c|}
\hline & \multicolumn{2}{|l|}{ Case } & \multicolumn{2}{|l|}{ Controls } & \multirow{2}{*}{$\begin{array}{l}\text { Unadjusted } \\
\mathrm{OR}^{\mathrm{a}}\end{array}$} & \multirow[t]{2}{*}{$95 \% \mathrm{Cl}$} \\
\hline & $n=2238$ & $\%$ & $n=8645$ & $\%$ & & \\
\hline Sex & & & & & NA & \\
\hline Female & 1359 & 60.7 & 5222 & 60.4 & & \\
\hline Male & 879 & 39.3 & 3423 & 39.6 & & \\
\hline Age, years & & & & & NA & \\
\hline $65-74$ & 765 & 34.2 & 3046 & 35.2 & & \\
\hline $75-84$ & 958 & 42.8 & 3800 & 44.0 & & \\
\hline$\geq 85$ & 515 & 23.0 & 1799 & 20.8 & & \\
\hline Index year & & & & & NA & \\
\hline 2003 & 175 & 7.8 & 682 & 7.9 & & \\
\hline 2004 & 214 & 9.6 & 836 & 9.7 & & \\
\hline 2005 & 211 & 9.4 & 817 & 9.5 & & \\
\hline 2006 & 218 & 9.7 & 855 & 9.9 & & \\
\hline 2007 & 257 & 11.5 & 1005 & 11.6 & & \\
\hline 2008 & 255 & 11.4 & 979 & 11.3 & & \\
\hline 2009 & 248 & 11.1 & 959 & 11.1 & & \\
\hline 2010 & 217 & 9.7 & 835 & 9.7 & & \\
\hline 2011 & 226 & 10.1 & 848 & 9.8 & & \\
\hline 2012 & 217 & 9.7 & 829 & 9.6 & & \\
\hline \multicolumn{7}{|l|}{ Comorbidities within 365 days before index date } \\
\hline Dementia & 197 & 8.8 & 550 & 6.4 & 1.40 & $1.18-1.67$ \\
\hline Epilepsy & 25 & 1.1 & 94 & 1.1 & 1.03 & $0.66-1.61$ \\
\hline Parkinson's disease & 165 & 7.4 & 333 & 3.9 & 1.98 & $1.64-2.40$ \\
\hline Cerebrovascular disease & 547 & 24.4 & 1758 & 20.3 & 1.27 & $1.13-1.42$ \\
\hline Diabetes & 629 & 28.1 & 2315 & 26.8 & 1.09 & $0.98-1.21$ \\
\hline Hypertension & 1296 & 57.9 & 5661 & 65.5 & 0.73 & $0.66-0.80$ \\
\hline Ischemia Heart Disease & 491 & 21.9 & 2341 & 27.1 & 0.76 & $0.68-0.85$ \\
\hline \multicolumn{7}{|l|}{ Medication exposure within 365 days before index date } \\
\hline Antipsychotics & 328 & 14.7 & 1011 & 11.7 & 1.31 & $1.14-1.50$ \\
\hline Antidepressants & 386 & 17.3 & 1089 & 12.6 & 1.45 & $1.28-1.65$ \\
\hline Anticonvulsants & 129 & 5.8 & 458 & 5.3 & 1.11 & $0.91-1.36$ \\
\hline Thiazide Diuretics & 235 & 10.5 & 1140 & 13.2 & 0.78 & $0.67-0.90$ \\
\hline \multirow[t]{2}{*}{ Opioids } & 378 & 16.9 & 687 & 8.0 & 2.38 & $2.07-2.73$ \\
\hline & Mean & SD & Mean & SD & & \\
\hline Age (years) & 77.7 & 7.2 & 77.3 & 7.0 & NA & \\
\hline Charlson Comorbidity Index score & 1.4 & 1.5 & 1.5 & 1.5 & 0.98 & $0.95-1.01$ \\
\hline Numbers of outpatient services in the 180 days before index date & 16.5 & 13.9 & 19.6 & 13.1 & 0.98 & $0.98-0.99$ \\
\hline
\end{tabular}

${ }^{\mathrm{a}}$ Significant results $(p<0.05)$ are in italicize

polypharmacy were all significantly more prominent than monotherapy of only one kind of BZD or only Z-drugs alone.

\section{Discussion}

In this population-based study, we observed that the current use of BZDs was associated with an increased risk of fall-related injuries requiring hospitalisation in older people, irrespective of dose levels (low, medium, or high) and elimination half-life (long-acting or shortacting). By contrast, the current use of Z-drugs, in particular at high dose levels increased this risk. With respect to polypharmacy, the use of more than one type of BZD significantly increased the risk, which reached 
Table 2 Unadjusted and adjusted odds ratios of BZD and Z-drug usage in the elderly patients hospitalised for fall injuries according to different type of exposure

\begin{tabular}{|c|c|c|c|c|c|c|c|c|}
\hline & \multicolumn{2}{|l|}{ Cases } & \multicolumn{2}{|l|}{ Controls } & \multicolumn{2}{|c|}{ Unadjusted } & \multicolumn{2}{|c|}{ Adjusted $^{\mathrm{ab}}$} \\
\hline & $n=2238$ & $\%$ & $n=8645$ & $\%$ & OR & $95 \% \mathrm{Cl}$ & OR & $95 \% \mathrm{Cl}$ \\
\hline \multicolumn{9}{|l|}{ BZDs } \\
\hline Current users & 672 & 30.0 & 2144 & 24.8 & 1.18 & $1.06-1.32$ & 1.32 & $1.17-1.50$ \\
\hline Indeterminate users & 160 & 7.2 & 879 & 10.2 & 0.69 & $0.57-0.83$ & 0.75 & $0.62-0.91$ \\
\hline Former users & 295 & 13.2 & 1519 & 17.6 & 0.73 & $0.63-0.84$ & 0.74 & $0.64-0.86$ \\
\hline Non-users & 1111 & 49.6 & 4103 & 47.5 & 1 & & 1 & \\
\hline \multicolumn{9}{|l|}{ Z-drugs } \\
\hline Current users & 224 & 10.0 & 721 & 8.3 & 1.23 & $1.05-1.45$ & 1.24 & $1.05-1.48$ \\
\hline Indeterminate users & 59 & 2.6 & 263 & 3.0 & 0.89 & $0.67-1.19$ & 0.87 & $0.64-1.18$ \\
\hline Former users & 141 & 6.3 & 572 & 6.6 & 0.98 & $0.81-1.18$ & 0.96 & $0.78-1.17$ \\
\hline Non- users & 1814 & 81.1 & 7089 & 82.0 & 1 & & 1 & \\
\hline
\end{tabular}

${ }^{a}$ Adjusted for dementia, Parkinson's disease, cerebrovascular disease, diabetes, hypertension, ischemia heart disease, antipsychotics, antidepressants, anticonvulsants, thiazide diuretics, opioids, Charlson comorbidity index, and number of outpatient services in the 180 days before the index date

${ }^{\mathrm{b}}$ Significant results $(p<0.05)$ are in italicize

the highest prominence when two or more types of BZD plus Z-drugs were used.

Our study results revealed that even low doses $(<0.3$ DDD) of BZDs were associated with an increased risk of fall-related injuries requiring hospitalisation among older people, and this risk correlated with an increase in the dose. A study [19] reported that a BZD daily dose higher than 0.75 DDD increased the risk of falls leading to femur fractures among subjects aged 55 years and older. The results of our previous study [13] and another study [14] demonstrated that a BZD daily dose higher than 0.3 DDD increased the risk of hip fractures in older people. These studies suggest that physicians should avoid prescribing BZDs to older people considering the high risk of falls even on low dose.
The 2002 Beers criteria [34] suggested that physicians should avoid prescribing long-acting BZDs to adults aged 65 years and older because they are potentially inappropriate medications. However, the 2012 and 2015 Beers criteria [35, 36] further suggested that the prescription of any BZD to older people is potentially inappropriate. Our data are consistent with those of previous studies $[13,14,18]$ and support the notion that short-acting BZDs are not safer than long-acting BZDs in older people.

Previous studies have reported that zolpidem increased the risk of falls [24] or fractures [25] in older people, our results also indicated that the current use of Z-drugs, in particular at high dose levels $(>0.6$ DDD) increased the risk of hospitalisation for fallrelated injuries in older people.

Table 3 Unadjusted and adjusted odds ratio of BZD and Z-drug usage in the elderly patients hospitalised for fall injuries according to dose levels among current users

\begin{tabular}{|c|c|c|c|c|c|c|c|c|}
\hline & \multicolumn{2}{|l|}{ Cases } & \multicolumn{2}{|l|}{ Controls } & \multicolumn{2}{|c|}{ Unadjusted } & \multicolumn{2}{|c|}{ Adjusted $^{\mathrm{ab}}$} \\
\hline & $n=2238$ & $\%$ & $n=8645$ & $\%$ & OR & $95 \%$ Cl & OR & $95 \% \mathrm{Cl}$ \\
\hline \multicolumn{9}{|l|}{ BZDs } \\
\hline High & 237 & 10.6 & 651 & 7.5 & 1.53 & $1.31-1.80$ & 1.75 & $1.47-2.08$ \\
\hline Medium & 196 & 8.8 & 639 & 7.4 & 1.30 & $1.10-1.54$ & 1.54 & $1.28-1.85$ \\
\hline Low & 239 & 10.7 & 854 & 9.9 & 1.17 & $1.00-1.37$ & 1.27 & $1.08-1.50$ \\
\hline Non-users & 1566 & 70.0 & 6501 & 75.2 & 1 & & 1 & \\
\hline \multicolumn{9}{|l|}{ Z-drugs } \\
\hline High & 203 & 9.1 & 609 & 7.0 & 1.34 & $1.13-1.58$ & 1.37 & $1.14-1.64$ \\
\hline Medium & 19 & 0.9 & 96 & 1.1 & 0.78 & $0.48-1.28$ & 0.77 & $0.46-1.28$ \\
\hline Low & 2 & 0.1 & 16 & 0.2 & 0.50 & $0.11-2.16$ & 0.46 & $0.10-2.08$ \\
\hline Non-users & 2014 & 90.0 & 7924 & 91.7 & 1 & & 1 & \\
\hline
\end{tabular}

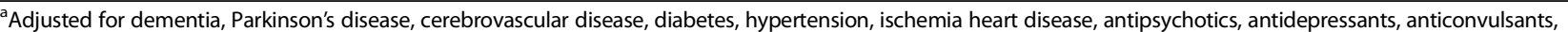
thiazide diuretics, opioids, Charlson comorbidity index, and number of outpatient services in the 180 days before the index date

${ }^{\mathrm{b}}$ Significant results $(p<0.05)$ are in italicize 
Table 4 Effects of BZD and Z-drug properties, half-life, and polypharmacy on the elderly patients hospitalised for fall injuries

\begin{tabular}{|c|c|c|c|c|c|c|c|c|}
\hline & \multicolumn{2}{|l|}{ Cases } & \multicolumn{2}{|l|}{ Controls } & \multicolumn{2}{|c|}{ Unadjusted } & \multicolumn{2}{|c|}{ Adjusted $^{\mathrm{ab}}$} \\
\hline & $n=2238$ & $\%$ & $n=8645$ & $\%$ & $\overline{\mathrm{OR}}$ & $95 \% \mathrm{Cl}$ & $\overline{\mathrm{OR}}$ & $95 \% \mathrm{Cl}$ \\
\hline \multicolumn{9}{|l|}{ Elimination half-life of BZD } \\
\hline Only long-acting BZD & 160 & 7.2 & 502 & 5.8 & 1.35 & $1.12-1.62$ & 1.41 & $1.16-1.71$ \\
\hline Only short-acting BZD & 213 & 9.5 & 692 & 8.0 & 1.29 & $1.10-1.52$ & 1.42 & $1.20-1.69$ \\
\hline Long- + short- acting BZD & 299 & 13.4 & 950 & 11.0 & 1.33 & $1.15-1.53$ & 1.61 & $1.37-1.89$ \\
\hline Non-users & 1566 & 70.0 & 6501 & 75.2 & 1 & & 1 & \\
\hline \multicolumn{9}{|l|}{ Polypharmacy of BZD and Z-drugs } \\
\hline Only one kind of BZD & 238 & 10.6 & 781 & 9.0 & 1.30 & $1.11-1.52$ & 1.40 & $1.19-1.65$ \\
\hline Two or more kinds of BZD & 309 & 13.8 & 979 & 11.3 & 1.35 & $1.17-1.55$ & 1.61 & $1.38-1.89$ \\
\hline Only Z-drugs & 99 & 4.4 & 337 & 3.9 & 1.26 & $1.00-1.59$ & 1.33 & $1.04-1.69$ \\
\hline One kind of BZD + Z-drugs & 34 & 1.5 & 89 & 1.0 & 1.61 & $1.08-2.40$ & 1.65 & $1.08-2.50$ \\
\hline Two or more kinds of BZD + Z-drugs & 91 & 4.1 & 295 & 3.4 & 1.33 & $1.04-1.70$ & 1.58 & $1.21-2.07$ \\
\hline Non-users & 1467 & 65.6 & 6164 & 71.3 & 1 & & 1 & \\
\hline
\end{tabular}

${ }^{a}$ Adjusted for dementia, Parkinson's disease, cerebrovascular disease, diabetes, hypertension, ischemia heart disease, antipsychotics, antidepressants, anticonvulsants, thiazide diuretics, opioids, Charlson comorbidity index, and number of outpatient services in the 180 days before the index date

${ }^{\mathrm{b}}$ Significant results $(p<0.05)$ are in italicize

In this study, we observed that the concomitant use of two or more types of BZD or any BZDs plus Z-drugs were significantly associated with the risk of hospitalisation for fall-related injuries among older people. Such definition of polypharmacy may differ from other studies $[26,37]$, which define it as the combined use of 5 or more drugs daily. Because such anxiolytic-hypnotic polypharmacy is common and has increased in Taiwan [27], more attention should be focused on the potential risk of falls in older people.

Two unexpected findings were noted in this study. First, the cases were less numbers of outpatient services in the 180 days before the index date. It is possible due to a bias in the selection of controls (e.g., some controls may have severe physical comorbidities which make them totally bed ridden and less prone to falls). Second, compared with BZDs non-users, indeterminate users and former users seemed to have lower risk of fallrelated injuries than non-users. Such results may be due to some uncontrolled confounding factor. However, the indeterminate and former users may have protected effects which suggest older people who stop BZDs may reduce the risk of fall-related hospitalisations. Several limitations should be considered before interpreting our results. First, since the patients with less severe fallrelated injuries may not have been recorded in the NHIRD, we used E-code to define the outcome. However, the E-code may not routinely be recorded by physicians in Taiwan and may have poor sensitivity. Such definition may underestimate or overestimate the fall risk of BZDs / Z-drugs to the elderly. Second, we defined BZDs and Z-drugs exposure within 1-30 days before the index date as "current use", which followed the classification of Ray et al. [31] in their earlier paper. However, they proposed the potentially serious misclassification in the study of the "acute effects" of BZDs and other drugs used intermittently upon studies of related injuries and suggested to track exposure on a day-by-day basis in future studies [38]. Third, although we used CCI and healthcare utilizations to control the comorbidities, some additional residual confounding factors should be considered such as vision problems, body mass index, physical activity, smoking, and alcohol use which may not be available from our data. Additionally, the functional abilities (gait speed, difficulties with activities of daily living) would be also important but not be available with administrative data. Forth, because the NHIRD only provided prescription records of drugs, we are unable to assert the exactness of each subject's medicating status, as noncompliance or "on a need basis" are also possible. Last, confounding by indication is another major bias in the observatory pharmacoepidemiological studies and may underestimate or overestimate the risk between BZDs/Z-drugs and fall in elderly people.

Despite several limitations listed above, our study has several strengths. The sample size used (NHIRD) was large, as it consists of a nationwide registry for medical claims data in Taiwan. In addition, ours is one of the few studies simultaneously examining the specific characteristics of BZD and Z-drug use among older people in a single study, with a focus on the type of exposure, daily dose, elimination half-life, and polypharmacy. Additionally, this is the first study to investigate the effects of polypharmacy with BZDs and Z-drugs on the risk of hospitalisation for fall-related injuries among older people. Consideration that Z-drugs are increasingly used 
as a common treatment choice for insomnia and the combined use of BZDs and Z-drugs is increasing in older people, the focus of this study is relevant for clinical decisions.

\section{Conclusion}

In conclusion, this study shows that different dose levels and half-lives of BZDs, a high dose of Z-drugs, and polypharmacy with BZDs and Z-drugs were associated with an increased risk of fall-related injury requiring hospitalisation in older people. Physicians should balance the risks and benefits when prescribing these drug regimens to older people considering the risk of falls.

\section{Abbreviations \\ AOR: adjusted odds ration; ATC: Anatomical Therapeutic Chemical; BZD: benzodiazepine; CCl: Charlson comorbidity index; Cl: confidential interval; DDD: defined daily dose; ICD-9-CM: International Classification of Disease, Ninth Revision, Clinical Modification; LHID: Longitudinal Health Insurance Database; MHW: Ministry of Health and Welfare; NHI: National Health Insurance; NHIRD: National Health Insurance Research Database; WHOCC: World Health Organization Collaborating Centre}

\section{Acknowledgments}

This study was based in part on data from the NHIRD provided by the National Health Insurance Administration, the Ministry of Health and Welfare and maintained by the National Health Research Institute. The interpretation and conclusions contained herein do not represent those of the Bureau of National Health Insurance, Department of Health or National Health Research Institutes.

\section{Availability of data materials}

The data of this study was available from the National Health Insurance Research Database (NHIRD) published by Taiwan National Health Insurance $(\mathrm{NHI})$ Bureau. Due to legal restrictions imposed by the government of Taiwan in relation to the "Personal Information Protection Act", data cannot be made publicly available. Requests for data can be sent as a formal proposal to the NHIRD (http:nhird.nhri.org.tw).

\section{Funding}

This study was funded by a grant from the Food and Drug Administration, Ministry of Health and Welfare, Taiwan (grant number MOHW104-FDA-D114-000632).

\section{Authors' contributions}

CMC designed and supervised the study, revision the manuscript. NWY contributed in the drafting and revising the manuscript. HJT perform the data analysis. PJC, HJT, CWH, and YWC were contributed to interpreting the research data. WIT and JH were contributed to revision the manuscript. All authors read and approved the final manuscript.

\section{Ethics approval and consent to participate}

This study was approved by the Institutional Review Board of Chang Gung Memorial Hospital (No.103-6020B). No informed consent was required from the subjects because the data were analysed anonymously.

\section{Consent for publication}

Not applicable.

\section{Competing interests}

The authors declare that they have no competing interests.

\section{Publisher's Note}

Springer Nature remains neutral with regard to jurisdictional claims in published maps and institutional affiliations.

\section{Author details}

'Department of Psychiatry, Chang Gung Memorial Hospital at Linkou and Chang Gung University, Taoyuan, Taiwan. ${ }^{2}$ Division of Rehabilitation \& Community Psychiatry, Department of Psychiatry, Chang Gung Memorial Hospital at Taoyuan, Taoyuan, Taiwan. ${ }^{3}$ Department of Pediatrics, Feinberg School of Medicine, Northwestern University, Chicago, IL, USA. ${ }^{4}$ Division of Biostatistics and Bioinformatics, Institute of Population Health Sciences, National Health Research Institutes, Miaoli, Taiwan. ${ }^{5}$ Division of Controlled Drugs, Taiwan Food and Drug Administration (TFDA), Ministry of Health and Welfare, Executive Yuan, Taipei, Taiwan.

Received: 12 October 2016 Accepted: 4 July 2017

Published online: 11 July 2017

\section{References}

1. WHO. Falls: http://www.who.int/mediacentre/factsheets/fs344/en/. Accessed 10 Sep 2016.

2. Masud T, Morris RO. Epidemiology of falls. Age Ageing. 2001;30(Suppl 4):3-7.

3. Alexander BH, Rivara FP, Wolf ME. The cost and frequency of hospitalization for fall-related injuries in older adults. Am J Public Health. 1992;82(7):1020-3.

4. Neutel $\mathrm{Cl}$, Perry S, Maxwell C. Medication use and risk of falls. Pharmacoepidemiol Drug Saf. 2002;11(2):97-104.

5. Kelly KD, Pickett W, Yiannakoulias N, Rowe BH, Schopflocher DP, Svenson L, et al. Medication use and falls in community-dwelling older persons. Age Ageing. 2003;32(5):503-9.

6. Leipzig RM, Cumming RG, Tinetti ME. Drugs and falls in older people: a systematic review and meta-analysis: I. Psychotropic drugs J Am Geriatr Soc. 1999:47(1):30-9.

7. Woolcott JC, Richardson KJ, Wiens MO, Patel B, Marin J, Khan KM, et al. Meta-analysis of the impact of 9 medication classes on falls in elderly persons. Arch Int Med. 2009;169(21):1952-60.

8. Hartikainen $\mathrm{S}$, Lonnroos $\mathrm{E}$, Louhivuori K. Medication as a risk factor for falls: critical systematic review. J Gerontol A Biol Sci Med Sci. 2007;62(10): 1172-81.

9. Olfson M, King M, Schoenbaum M. Benzodiazepine use in the United States. JAMA Psychiatry. 2015;72(2):136-42.

10. Brett J, Murnion B. Management of benzodiazepine misuse and dependence. Aust Prescr. 2015;38(5):152-5.

11. Chang CM, Chen MJ, Tsai CY, Ho LH, Hsieh HL, Chau YL, et al. Medical conditions and medications as risk factors of falls in the inpatient older people: a case-control study. Int J Geriatr Psychiatry. 2011;26(6):602-7.

12. Pariente A, Dartigues JF, Benichou J, Letenneur L, Moore N, Fourrier-Reglat A. Benzodiazepines and injurious falls in community dwelling elders. Drugs Aging. 2008;25(1):61-70

13. Chang CM, Wu EC, Chang IS, Lin KM. Benzodiazepine and risk of hip fractures in older people: a nested case-control study in Taiwan. Am J Geriatr Psychiatry. 16(8):686-92.

14. Wang PS, Bohn RL, Glynn RJ, Mogun H, Avorn J. Hazardous benzodiazepine regimens in the elderly: effects of half-life, dosage, and duration on risk of hip fracture. Am J Psychiatry. 2001;158(6):892-8.

15. Chang CM, Wu EC, Chen CY, Wu KY, Liang HY, Chau YL, et al. Psychotropic drugs and risk of motor vehicle accidents: a population-based case-control study. Brit J Clin Pharmacology. 2013;75(4):1125-33.

16. Ballokova A, Peel NM, Fialova D, Scott IA, Gray LC, Hubbard RE. Use of benzodiazepines and association with falls in older people admitted to hospital: a prospective cohort study. Drugs Aging. 2014;31(4):299-310.

17. van Strien AM, Koek HL, van Marum RJ, Emmelot-Vonk MH. Psychotropic medications, including short acting benzodiazepines, strongly increase the frequency of falls in elderly. Maturitas. 2013;74(4):357-62.

18. de Vries OJ, Peeters G, Elders P, Sonnenberg C, Muller M, Deeg DJ, et al. The elimination half-life of benzodiazepines and fall risk: two prospective observational studies. Age Ageing. 2013;42(6):764-70.

19. Herings RM, Stricker BH, de Boer A, Bakker A, Sturmans F. Benzodiazepines and the risk of falling leading to femur fractures. Dosage more important than elimination half-life. Arch Int Med. 1995;155(16):1801-7.

20. Darcourt G, Pringuey D, Salliere D, Lavoisy J. The safety and tolerability of zolpidem-an update. J Psychopharmacol. 1999;13(1):81-93.

21. Huang WF, Lai IC. Patterns of sleep-related medications prescribed to elderly outpatients with insomnia in Taiwan. Drugs Aging. 2005;22(11):957-65. 
22. Hsiao FY, Hsieh PH, Gau CS. Ten-year trend in prescriptions of z-hypnotics among the elderly: a nationwide, cross-sectional study in Taiwan. J Clin Geronto Geriatr. 2013;4(2):37-41.

23. Victorri-Vigneau C, Dailly E, Veyrac G, Jolliet P. Evidence of zolpidem abuse and dependence: results of the French Centre for Evaluation and Information on Pharmacodependence (CEIP) network survey. Brit J Clin Pharmacology. 2007;64(2):198-209.

24. Diem SJ, Ewing SK, Stone KL, Ancoli-Israel S, Redline S, Ensrud KE, et al. Use of non-benzodiazepine sedative hypnotics and risk of falls in older men. J Geronto Geriatr Res. 2014;3(3):158.

25. Wang PS, Bohn RL, Glynn RJ, Mogun H, Avorn J. Zolpidem use and hip fractures in older people. J Am Geriatr Soc. 2001;49(12):1685-90.

26. Richardson K, Bennett K, Kenny RA. Polypharmacy including falls risk-increasing medications and subsequent falls in community-dwelling middle-aged and older adults. Age Ageing. 2015:44(1):90-6.

27. Wang L, Chen YC, Chen CK, Chou WJ, Chou MC. Trends in anxiolytic-hypnotic use and polypharmacy in Taiwan, 2002-2009: a nationwide, population-based survey. Psychiatr Serv. 2014;65(2):208-14.

28. Hsu J, Lin JJ, Tsay WI. Analysis of drug abuse data reported by medical institutions in Taiwan from 2002 to 2011. J Food Drug Anal. 2014;22(2):169-77.

29. Fang SY, Chen CY, Chang IS, et al. Predictors of the incidence and discontinuation of long-term use of benzodiazepines: a population-based study. Drug Alcohol Depend. 2009;10:140-6.

30. National Health Insurance Research Database (NHIRD). Introduction to the National Health Insurance Research Database (NHIRD), Taiwan. 2010; http://nhird.nhri.org.tw/en/. Accessed 10 Sep 2016

31. Ray WA, Griffin MR, Downey W. Benzodiazepines of long and short elimination half-life and the risk of hip fracture. JAMA. 1989;262(23):3303-7.

32. WHO. ATC/DDD Index. http://www.whocc.no/atc_ddd_index/. Accessed 10 Sep 2016.

33. Charlson ME, Pompei P, Ales KL, MacKenzie CR. A new method of classifying prognostic comorbidity in longitudinal studies: development and validation. J Chronic Dis. 1987:40:373-83.

34. Fick DM, Cooper JW, Wade WE, Waller JL, Maclean JR, Beers MH. Updating the beers criteria for potentially inappropriate medication use in older adults: results of a US consensus panel of experts. Arch Int Med. 2003: 163(22):2716-24

35. Campanelli CM. American Geriatrics Society updated beers criteria for potentially inappropriate medication use in older adults: the American Geriatrics Society 2012 beers criteria update expert panel. J Am Geriatr Soc. 2012;60(4):616

36. Radcliff S, Yue J, Rocco G, Aiello SE, Ickowicz E, Hurd Z, Samuel MJ, Beers MH. American Geriatrics Society 2015 updated beers criteria for potentially inappropriate medication use in older adults. J Am Geriatr Soc. 2015:63(11):2227-46.

37. Lai SW, Liao KF, Liao CC, Muo CH, Liu CS, Sung FC. Polypharmacy correlates with increased risk for hip fracture in the elderly: a population-based study. Medicine. 2010;89(5):295-9.

38. Ray WA, Thapa PB, Gideon P. Misclassification of current benzodiazepine exposure by use of a single baseline measurement and its effects upon studies of injuries. Pharmacoepid Drug Saf. 2002;11(8):663-9.

\section{Submit your next manuscript to BioMed Central and we will help you at every step:}

- We accept pre-submission inquiries

- Our selector tool helps you to find the most relevant journal

- We provide round the clock customer support

- Convenient online submission

- Thorough peer review

- Inclusion in PubMed and all major indexing services

- Maximum visibility for your research

Submit your manuscript at www.biomedcentral.com/submit

) Biomed Central 